\title{
Quantificação de doenças pós-colheita em acessos de goiabeira cultivados em sistema orgânico ${ }^{1}$
}

\author{
Juliana Altafin Galli², Ivan Herman Fischer ${ }^{3}$, \\ Maria Cecília de Arruda Palharini ${ }^{3}$, Marcos Doniseti Michelotto
}

\begin{abstract}
Quantification of postharvest diseases of guava accessions cultivated in organic system

Among the postharvest diseases that occur in guava, anthracnose, black spot and stem-end rot are the most common. The incidence and diversity of these diseases depend on the host genotype. This study aimed to identify and quantify the postharvest diseases of guava accessions cultivated in organic system. For the characterization of diseases, 30 fruits from 48 accessions were evaluated in a completely randomized design. Anthracnose and black spot were the most frequent postharvest diseases in guava. The Vermelha Redonda (Shimoda), L5P21, L3P12, EEF-3, IAC-4 - Unesp and Monte Alto - Comum 1 accessions stood out with the lowest incidence of anthracnose and black spot, while L4P13, L1P2 and Creme Arredondada (Unesp) showed the highest incidence of anthracnose, black spot and stem-end rot. The Fusicoccum sp., Phomopsis sp., Phoma sp., Dothiorella sp. and Lasiodiplodia sp. pathogens were identified as the main responsible for causing stem-end rot symptoms.
\end{abstract}

KEY-WORDS: Psidium guajava; anthracnose; black spot; stem-end rot.

\section{INTRODUÇÃO}

Entre as frutas tropicais, a goiaba ocupa lugar de destaque por sua excelente qualidade, atribuída a quatro fatores principais: elevado valor nutritivo, excelentes propriedades organolépticas, alto rendimento por hectare e polpa com elevada qualidade industrial.

A expansão da comercialização de frutos está intrinsecamente ligada à qualidade destes e ao aumento de sua vida pós-colheita. Dentre os principais fatores responsáveis pela depreciação dos frutos e redução de sua vida útil estão as doenças que comprometem a sua aparência e alteram a sua consistência, cor e sabor (Manica et al. 2000, Barkai-Golan 2001).

\section{RESUMO}

Dentre as doenças que ocorrem na pós-colheita da goiaba, destacam-se a antracnose, a pinta preta e as podridões pedunculares. A incidência e a diversidade dessas doenças dependem do genótipo do hospedeiro. Este trabalho objetivou identificar e quantificar as doenças pós-colheita de acessos de goiabeira cultivados em sistema orgânico. Para a caracterização das doenças, avaliaram-se 30 frutos de 48 acessos, em delineamento inteiramente casualizado. A antracnose e a pinta preta foram as doenças pós-colheita mais frequentes na goiaba. Os acessos Vermelha Redonda (Shimoda), L5P21, L3P12, EEF-3, IAC-4 - Unesp e Monte Alto - Comum 1 destacaram-se por apresentarem as menores incidências de antracnose e pinta preta, enquanto L4P13, L1P2 e Creme Arredondada (Unesp) foram os que apresentaram as maiores incidências de antracnose, pinta preta e podridão peduncular. Os patógenos Fusicoccum sp., Phomopsis sp., Phoma sp., Dothiorella sp. e Lasiodiplodia sp. foram identificados como causadores de sintomas da podridão peduncular.

PALAVRAS-CHAVE: Psidium guajava; antracnose; pinta preta; podridão peduncular.

Das doenças que ocorrem na pós-colheita da goiaba, as mais importantes são as quiescentes, cuja infecção pode ocorrer a qualquer momento, desde o início da formação do fruto, ainda no campo, até sua manipulação e armazenamento pós-colheita. Dentre essas doenças, destacam-se a antracnose (Colletotrichum gloeosporioides e C. acutatum), pinta preta (Guignardia psidii) e podridões pedunculares (Fusicoccum sp., Phoma psidii, Phomopsis spp.). Doenças de infecções imediatas incluem as podridões de Rhizopus stolonifer, Pestalotia psidii e Aspergillus niger. A epidemiologia dessas doenças é muito distinta, com implicações importantes para o seu controle (Amorim et al. 2009, Fischer et al. 2011).

1. Trabalho recebido em jan./2015 e aceito para publicação em jun./2015 (http://dx.doi.org/10.1590/1983-40632015v4533713).

2. Agência Paulista de Tecnologia dos Agronegócios (APTA), Polo Regional Centro-Norte, Pindorama, SP, Brasil.

E-mails: julianagalli@apta.sp.gov.br, michelotto@apta.sp.gov.br.

3. Agência Paulista de Tecnologia dos Agronegócios (APTA), Polo Regional Centro-Oeste, Bauru, SP, Brasil.

E-mails: ihfische@apta.sp.gov.br, mcarruda@apta.sp.gov.br. 
Os sintomas da antracnose caracterizam-se, inicialmente, por manchas necróticas pequenas, deprimidas e de coloração marrom clara, na superfície dos frutos. Com a evolução da doença, as lesões tornam-se irregulares, mais deprimidas, chegando a atingir 1-1,5 cm de diâmetro. Sob condições de alta umidade, uma massa de esporos alaranjada se forma sobre o centro das lesões (Piccinin et al. 2005).

Os sintomas da pinta preta constituem-se de pontos amarelados deprimidos, os quais evoluem rapidamente na superfície dos frutos, de forma concêntrica, tornando-se lesões de coloração preta esverdeada, que podem apresentar, na superfície, frutificações do fungo, geralmente conidiomas picnidiais. Essas lesões, de aproximadamente 1-2,5 cm de diâmetro, ocorrem em qualquer área do fruto, podendo, posteriormente, coalescer (Tozetto \& Ribeiro 1993, Junqueira \& Costa 2002).

Já as podridões pedunculares caracterizam-se por lesões escuras, com bordos bem definidos, que se iniciam, geralmente, a partir do ápice e avançam para o pedúnculo, tomando todo o fruto (Junqueira \& Costa 2002).

É recomendado que o controle de doenças pós-colheita em goiaba seja efetuado ainda no campo, por meio de poda de ramos infectados e pela aplicação de pulverizações preventivas com fungicidas (Junqueira \& Costa 2002). Contudo, o uso crescente e indiscriminado de agrotóxicos na cultura de espécies frutíferas tem colocado em risco a saúde de consumidores e produtores rurais, além de gerar sérios problemas ambientais (Pinheiro 2006).

$\mathrm{O}$ emprego de variedades resistentes às principais doenças da goiabeira poderia contribuir para a redução de danos e perdas, inclusive na pós-colheita. A utilização de variedades resistentes é especialmente importante nos pomares cultivados em sistema orgânico. Dessa maneira, torna-se importante conhecer o padrão de resistência/suscetibilidade de acessos de goiabeira, em relação às doenças pós-colheita.

Diante do exposto, o presente trabalho objetivou identificar e quantificar as doenças pós-colheita de acessos de goiabeira cultivados em sistema orgânico.

\section{MATERIAL E MÉTODOS}

O experimento foi desenvolvido na Agência Paulista de Tecnologia dos Agronegócios (APTA), Polo Regional Centro-Norte, em Pindorama (SP) $\left(21^{\circ} 13^{\prime} \mathrm{S}\right.$ e $\left.48^{\circ} 55^{\prime} \mathrm{W}\right)$, onde a temperatura média anual é de $22,8{ }^{\circ} \mathrm{C}$, a precipitação média anual de $1.390,3 \mathrm{~mm}$ e a umidade relativa média anual de 71,6 \%. Conforme a classificação de Köppen, o clima é do tipo Aw, definido como tropical úmido, com estação chuvosa no verão e seca no inverno.

Utilizaram-se 85 acessos do Banco Ativo de Germoplasma de goiabeira, com 15 anos de idade, sendo três plantas/acesso, cultivadas em sistema orgânico, em espaçamento $6 \mathrm{~m}$ x $5 \mathrm{~m}$. Para alguns acessos, não foi possível a coleta de frutos para todas as análises propostas, devido à baixa produção ou ausência de frutos. Por isso, foram avaliados somente 48 acessos. Mediante o resultado da análise de solo, foi realizada uma aplicação em superfície de 1,5 ton. ha ${ }^{-1}$ de calcário dolomítico, na área total, e $20 \mathrm{~L}$ de torta de filtro, ao redor do tronco de cada planta. O controle de plantas espontâneas foi realizado por roçadeira tratorizada e capinas manuais.

Para a caracterização das doenças pós-colheita, foram utilizados 30 frutos de 48 acessos. Os frutos foram obtidos de três plantas (repetições), com uma amostra de 10 frutos cada. Após a colheita, ocorrida entre janeiro e março de 2014, os frutos foram transportados, no mesmo dia, até o laboratório do Polo Regional Centro-Oeste (APTA), em Bauru (SP). Os frutos foram individualizados em bandejas plásticas, permanecendo por oito dias a $25 \pm 1{ }^{\circ} \mathrm{C}$ e $70-80 \%$ de umidade relativa. A incidência (porcentagem de frutos com doenças pós-colheita) foi visualmente avaliada após 2, 4, 6 e 8 dias da colheita.

Os fungos foram identificados com o auxílio de microscópio óptico e chave dicotômica (Barnett \& Hunter 1998), com posterior isolamento em meio de cultura BDA, em caso de dúvida na identificação do fungo. A patogenicidade dos fungos foi confirmada mediante a inoculação em frutos sadios de goiaba (Fischer et al. 2011). Após a obtenção dos dados de incidência, foi calculada a área abaixo da curva de progresso da doença (AACPD), de acordo com Campbell \& Maden (1990).

Os dados de incidência e AACPD das doenças observadas foram submetidos à análise de variância, utilizando-se delineamento experimental inteiramente casualizado. As médias dos acessos de goiabeira para cada doença foram comparadas pelo teste de Scott-Knott, a 5\%.

\section{RESULTADOS E DISCUSSÃO}

As principais doenças observadas nos acessos de goiabeira foram a antracnose, pinta preta e podridão 
peduncular e, em menores incidências, as podridões por Pestalotia e Mucor. Diferenças significativas entre os acessos foram observadas para essas principais doenças, à exceção da podridão por Pestalotia (Tabelas 1 e 2).
Para a antracnose, os acessos de goiabeira com menores incidências, considerando-se simultaneamente a AACPD e a incidência final, foram: Vermelha Redonda (Shimoda), L4P16, Patillo, L7P26, Monte

Tabela 1. Área abaixo da curva de progresso da doença (AACPD) de podridões pós-colheita, após oito dias de armazenamento dos frutos a $25 \pm 1{ }^{\circ} \mathrm{C}$ e $70-80 \%$ de umidade relativa (Bauru, SP, 2014).

\begin{tabular}{|c|c|c|c|c|c|c|c|c|c|c|}
\hline \multirow{3}{*}{ Acessos } & \multicolumn{10}{|c|}{$\mathrm{AACPD}^{1}$} \\
\hline & \multicolumn{2}{|c|}{ Antracnose } & \multicolumn{2}{|c|}{$\begin{array}{l}\text { Pinta } \\
\text { preta }\end{array}$} & \multicolumn{2}{|c|}{$\begin{array}{l}\text { Podridão } \\
\text { peduncular }\end{array}$} & \multicolumn{2}{|c|}{$\begin{array}{c}\text { Podridão por } \\
\text { Pestalotia }\end{array}$} & \multicolumn{2}{|c|}{$\begin{array}{c}\text { Podridão por } \\
\text { Mucor }\end{array}$} \\
\hline & 3,8 & $\mathrm{a}^{2}$ & 12,5 & $\mathrm{c}$ & 12,1 & $\mathrm{~b}$ & 0,0 & $\mathrm{a}$ & 0,0 & $\bar{a}$ \\
\hline Vermelha Redonda (Shimoda) & 4,8 & $\mathrm{a}$ & 18,1 & $\mathrm{c}$ & 4,3 & a & 7,2 & $\mathrm{a}$ & 3,7 & $\mathrm{~b}$ \\
\hline L7P26 & 5,2 & $\mathrm{a}$ & 11,0 & $\mathrm{c}$ & 10,8 & $\mathrm{~b}$ & 2,0 & $\mathrm{a}$ & 0,0 & $\mathrm{a}$ \\
\hline L4P16 & 6,2 & $\mathrm{a}$ & 33,3 & $\mathrm{~d}$ & 3,2 & $\mathrm{a}$ & 0,0 & $\mathrm{a}$ & 3,3 & $\mathrm{~b}$ \\
\hline Monte Alto - Comum 1 & 7,1 & $\mathrm{a}$ & 9,0 & $\mathrm{~b}$ & 2,9 & $\mathrm{a}$ & 0,0 & $\mathrm{a}$ & 0,0 & $\mathrm{a}$ \\
\hline EEF-3 & 7,3 & $\mathrm{a}$ & 1,0 & $\mathrm{a}$ & 7,0 & $\mathrm{~b}$ & 0,0 & $\mathrm{a}$ & 0,0 & $\mathrm{a}$ \\
\hline Kioshi3 & 7,4 & $\mathrm{a}$ & 13,7 & $\mathrm{c}$ & 9,3 & $\mathrm{~b}$ & 0,0 & $\mathrm{a}$ & 1,4 & $\mathrm{a}$ \\
\hline Ogawa x Kumagai & 7,7 & $\mathrm{a}$ & 19,1 & $\mathrm{c}$ & 11,3 & $\mathrm{~b}$ & 0,0 & $\mathrm{a}$ & 0,3 & $\mathrm{a}$ \\
\hline L3P9 & 8,1 & $\mathrm{a}$ & 31,6 & $\mathrm{~d}$ & 3,9 & $\mathrm{a}$ & 1,7 & $\mathrm{a}$ & 1,3 & $\mathrm{a}$ \\
\hline L5P20 & 10,0 & $\mathrm{~b}$ & 11,0 & $\mathrm{c}$ & 3,3 & $\mathrm{a}$ & 1,0 & $\mathrm{a}$ & 2,7 & $\mathrm{~b}$ \\
\hline L4P13 & 10,0 & $\mathrm{~b}$ & 14,3 & $\mathrm{c}$ & 12,3 & $\mathrm{~b}$ & 3,0 & $\mathrm{a}$ & 2,0 & $\mathrm{~b}$ \\
\hline IAC-4 - Unesp & 10,0 & $\mathrm{~b}$ & 29,0 & $\mathrm{~d}$ & 3,3 & $\mathrm{a}$ & 0,5 & $\mathrm{a}$ & 0,0 & $\mathrm{a}$ \\
\hline Monte Alto - Branca & 10,5 & $\mathrm{~b}$ & 30,2 & d & 6,0 & $\mathrm{a}$ & 1,7 & $\mathrm{a}$ & 5,2 & $\mathrm{~b}$ \\
\hline Rubi Supreme & 10,6 & $\mathrm{~b}$ & 5,4 & $\mathrm{~b}$ & 10,7 & $\mathrm{~b}$ & 1,7 & $\mathrm{a}$ & 1,7 & $\mathrm{~b}$ \\
\hline L2P4 & 11,3 & $\mathrm{~b}$ & 6,0 & $\mathrm{~b}$ & 18,0 & $\mathrm{~b}$ & 2,0 & $\mathrm{a}$ & 0,0 & $\mathrm{a}$ \\
\hline Webber Supreme & 11,5 & $\mathrm{~b}$ & 0,0 & $\mathrm{a}$ & 6,7 & $\mathrm{~b}$ & 0,0 & $\mathrm{a}$ & 1,9 & $\mathrm{~b}$ \\
\hline Indiana - Unesp & 12,0 & $b$ & 7,9 & $\mathrm{~b}$ & 6,2 & $\mathrm{a}$ & 0,0 & $\mathrm{a}$ & 1,7 & $\mathrm{~b}$ \\
\hline FAO - Unesp & 12,3 & $\mathrm{~b}$ & 1,3 & $\mathrm{a}$ & 3,0 & $\mathrm{a}$ & 0,0 & $\mathrm{a}$ & 2,7 & $\mathrm{~b}$ \\
\hline L3P12 & 12,8 & $\mathrm{~b}$ & 1,3 & $\mathrm{a}$ & 3,8 & $\mathrm{a}$ & 2,0 & $\mathrm{a}$ & 0,0 & $\mathrm{a}$ \\
\hline L2P6 & 13,3 & $\mathrm{~b}$ & 18,0 & $\mathrm{c}$ & 3,0 & $\mathrm{a}$ & 1,3 & $\mathrm{a}$ & 0,0 & $\mathrm{a}$ \\
\hline L8P32B & 13,3 & $\mathrm{~b}$ & 30,7 & $\mathrm{~d}$ & 8,0 & $\mathrm{~b}$ & 0,7 & $\mathrm{a}$ & 3,3 & $\mathrm{~b}$ \\
\hline Kumagai Branca & 13,3 & $\mathrm{~b}$ & 17,6 & $\mathrm{c}$ & 4,3 & $\mathrm{a}$ & 0,0 & $\mathrm{a}$ & 3,3 & $\mathrm{~b}$ \\
\hline Rica - J-2 & 13,9 & $\mathrm{~b}$ & 2,9 & $\mathrm{a}$ & 12,3 & $\mathrm{~b}$ & 0,0 & $\mathrm{a}$ & 0,0 & $\mathrm{a}$ \\
\hline L8P32A & 14,7 & $\mathrm{~b}$ & 7,3 & $\mathrm{~b}$ & 17,7 & $\mathrm{~b}$ & 0,3 & $\mathrm{a}$ & 2,0 & $\mathrm{~b}$ \\
\hline L5P21 & 15,7 & $\mathrm{~b}$ & 0,3 & $\mathrm{a}$ & 4,0 & $\mathrm{a}$ & 0,3 & $\mathrm{a}$ & 0,0 & $\mathrm{a}$ \\
\hline L5P18 & 17,5 & $\mathrm{c}$ & 15,0 & $\mathrm{c}$ & 9,4 & $\mathrm{~b}$ & 0,0 & $\mathrm{a}$ & 0,0 & $\mathrm{a}$ \\
\hline L6P22 & 18,0 & $\mathrm{c}$ & 0,7 & $\mathrm{a}$ & 5,7 & $\mathrm{a}$ & 3,0 & $\mathrm{a}$ & 0,0 & $\mathrm{a}$ \\
\hline L3P10 & 18,0 & $\mathrm{c}$ & 14,6 & $\mathrm{c}$ & 5,3 & $\mathrm{a}$ & 0,0 & $\mathrm{a}$ & 0,0 & $\mathrm{a}$ \\
\hline L6P23 & 18,9 & $\mathrm{c}$ & 19,4 & $\mathrm{c}$ & 3,2 & $\mathrm{a}$ & 1,3 & $\mathrm{a}$ & 4,2 & $\mathrm{~b}$ \\
\hline Ogawa3 & 19,6 & $\mathrm{c}$ & 4,5 & $\mathrm{~b}$ & 8,5 & $\mathrm{~b}$ & 0,0 & $\mathrm{a}$ & 0,0 & $\mathrm{a}$ \\
\hline Ogawa1 & 19,9 & c & 8,7 & $\mathrm{~b}$ & 5,3 & $\mathrm{a}$ & 0,0 & $\mathrm{a}$ & 0,0 & $\mathrm{a}$ \\
\hline L8P31 & 20,7 & c & 13,3 & $\mathrm{c}$ & 6,3 & $\mathrm{~b}$ & 0,7 & $\mathrm{a}$ & 0,0 & $\mathrm{a}$ \\
\hline L1P3 & 20,9 & $\mathrm{c}$ & 5,5 & b & 6,7 & $\mathrm{~b}$ & 0,0 & $\mathrm{a}$ & 0,0 & $\mathrm{a}$ \\
\hline L3P7 & 21,4 & $\mathrm{c}$ & 7,8 & $\mathrm{~b}$ & 12,5 & $\mathrm{~b}$ & 1,9 & $\mathrm{a}$ & 0,8 & $\mathrm{a}$ \\
\hline L4P17 & 23,0 & $\mathrm{c}$ & 11,8 & $\mathrm{c}$ & 8,1 & $\mathrm{~b}$ & 0,0 & $\mathrm{a}$ & 1,7 & $\mathrm{~b}$ \\
\hline Creme Arredondada (Unesp) & 23,7 & $\mathrm{c}$ & 19,0 & $\mathrm{c}$ & 9,4 & $\mathrm{~b}$ & 1,1 & $\mathrm{a}$ & 0,0 & $\mathrm{a}$ \\
\hline Kioshi2 & 23,7 & $\mathrm{c}$ & 23,4 & $\mathrm{~d}$ & 3,8 & $\mathrm{a}$ & 1,7 & $\mathrm{a}$ & 0,3 & a \\
\hline L4P15 & 24,4 & $\mathrm{c}$ & 17,0 & $\mathrm{c}$ & 4,9 & $\mathrm{a}$ & 0,3 & $\mathrm{a}$ & 0,0 & a \\
\hline Goiaba Polpa Amarela & 25,2 & $\mathrm{c}$ & 21,1 & $\mathrm{c}$ & 7,6 & $\mathrm{~b}$ & 2,0 & $\mathrm{a}$ & 0,0 & a \\
\hline Kioshi1 & 28,3 & $\mathrm{~d}$ & 28,3 & $\mathrm{~d}$ & 8,0 & $\mathrm{~b}$ & 0,0 & $\mathrm{a}$ & 0,0 & a \\
\hline L2P5 & 30,2 & $d$ & 20,8 & $\mathrm{c}$ & 9,5 & $\mathrm{~b}$ & 0,0 & $\mathrm{a}$ & 0,0 & $\mathrm{a}$ \\
\hline Vermelha Perfumada & 31,4 & d & 21,4 & $\mathrm{c}$ & 1,9 & $\mathrm{a}$ & 0,3 & $\mathrm{a}$ & 0,0 & $\mathrm{a}$ \\
\hline IAC-4 - Cica & 31,7 & d & 26,9 & $\mathrm{~d}$ & 8,3 & $\mathrm{~b}$ & 1,0 & $\mathrm{a}$ & 0,0 & a \\
\hline Campos & 32,0 & $d$ & 21,0 & $\mathrm{c}$ & 9,0 & $\mathrm{~b}$ & 0,0 & $\mathrm{a}$ & 0,0 & $\mathrm{a}$ \\
\hline L5P19 & 32,7 & d & 25,9 & $\mathrm{~d}$ & 2,2 & $\mathrm{a}$ & 0,3 & $\mathrm{a}$ & 0,0 & a \\
\hline L4P14 & 32,9 & d & 15,1 & $\mathrm{c}$ & 2,0 & $\mathrm{a}$ & 0,0 & $\mathrm{a}$ & 6,7 & $\mathrm{~b}$ \\
\hline Taquaritinga Comum & 33,3 & $\mathrm{~d}$ & 18,0 & $\mathrm{c}$ & 3,7 & $\mathrm{a}$ & 1,3 & $\mathrm{a}$ & 1,0 & $\mathrm{a}$ \\
\hline L1P2 & 40,7 & $\mathrm{~d}$ & 21,8 & $\mathrm{c}$ & 10,0 & $\mathrm{~b}$ & 0,0 & $\mathrm{a}$ & 0,0 & $\mathrm{a}$ \\
\hline $\mathrm{F}$ (acessos) & \multicolumn{2}{|c|}{$6,8^{* *}$} & \multicolumn{2}{|c|}{$10,1 * *$} & \multicolumn{2}{|c|}{$1,6^{*}$} & \multicolumn{2}{|c|}{1,4} & \multirow{2}{*}{\multicolumn{2}{|c|}{$\frac{1,8^{* *}}{35.4}$}} \\
\hline $\mathrm{CV}(\%)$ & 17 & & 19 & & 29 , & & 34, & & & \\
\hline
\end{tabular}

${ }^{1}$ AACPD: média de três repetições de 10 frutos cada, calculada a partir de quatro avaliações, em intervalos de dois dias cada. ${ }^{2}$ Dados seguidos pela mesma letra, na coluna, não diferem entre si, a $5 \%$, pelo teste de Scott-Knott. * Significativo a $5 \%$. * Significativo a $1 \%$. A análise estatística foi realizada com os dados transformados em $\sqrt{\mathrm{x}}+1$. 
Alto - Comum 1 e EEF-3, sem diferir significativamente entre si e com incidências abaixo de $35 \%$ (Tabelas 1 e 2). Os acessos Campos, L1P2 e Taquaritinga Comum apresentaram $100 \%$ de incidência, evidenciando a maior suscetibilidade dos mesmos. Para a pinta preta, os acessos menos afetados foram: Webber Supreme, EEF-3, L5P21, L3P12, L6P22 e FAO - Unesp, sem diferenças significativas e com

Tabela 2. Porcentagem de incidência de podridões pós-colheita, após oito dias de armazenamento dos frutos a $25 \pm 1{ }^{\circ} \mathrm{C}$ e $70-80 \%$ de umidade relativa (Bauru, SP, 2014).

\begin{tabular}{|c|c|c|c|c|c|c|c|c|c|c|}
\hline \multirow{3}{*}{$\begin{array}{c}\text { Acessos } \\
\text { Vermelha Redonda (Shimoda) }\end{array}$} & \multicolumn{10}{|c|}{ Incidência (\%) } \\
\hline & \multicolumn{2}{|c|}{ Antracnose } & \multicolumn{2}{|c|}{$\begin{array}{l}\text { Pinta } \\
\text { preta }\end{array}$} & \multicolumn{2}{|c|}{$\begin{array}{l}\text { Podridão } \\
\text { peduncular }\end{array}$} & \multicolumn{2}{|c|}{$\begin{array}{c}\text { Podridão por } \\
\text { Pestalotia }\end{array}$} & \multicolumn{2}{|c|}{$\begin{array}{c}\text { Podridão por } \\
\text { Mucor }\end{array}$} \\
\hline & 12,2 & $\mathrm{a}^{1}$ & 55,6 & $\mathrm{c}$ & 18,9 & $\bar{a}$ & 25,6 & $\bar{a}$ & 12,2 & $\mathrm{~b}$ \\
\hline L4P16 & 18,1 & $\mathrm{a}$ & 85,9 & $\mathrm{~d}$ & 25,2 & $\mathrm{~b}$ & 0,0 & $\mathrm{a}$ & 11,1 & $\mathrm{~b}$ \\
\hline Patillo & 20,8 & $\mathrm{a}$ & 41,7 & $\mathrm{c}$ & 45,8 & $\mathrm{~b}$ & 0,0 & $\mathrm{a}$ & 0,0 & $\mathrm{a}$ \\
\hline L7P26 & 25,6 & $\mathrm{a}$ & 56,7 & $\mathrm{c}$ & 32,2 & $\mathrm{~b}$ & 6,7 & $\mathrm{a}$ & 0,0 & $\mathrm{a}$ \\
\hline Monte Alto - Comum 1 & 28,6 & $\mathrm{a}$ & 47,6 & $\mathrm{c}$ & 9,5 & $\mathrm{a}$ & 0,0 & $\mathrm{a}$ & 0,0 & $\mathrm{a}$ \\
\hline EEF-3 & 33,3 & $\mathrm{a}$ & 3,3 & $\mathrm{a}$ & 50,0 & $\mathrm{~b}$ & 0,0 & a & 0,0 & $\mathrm{a}$ \\
\hline IAC-4 - Unesp & 33,3 & $\mathrm{a}$ & 90,5 & d & 14,3 & $\mathrm{a}$ & 4,8 & a & 0,0 & $\mathrm{a}$ \\
\hline Kioshi3 & 36,7 & $\mathrm{~b}$ & 46,7 & $\mathrm{c}$ & 22,2 & $\mathrm{a}$ & 0,0 & $\mathrm{a}$ & 2,8 & $\mathrm{a}$ \\
\hline Ogawa x Kumagai & 43,9 & b & 68,3 & $\mathrm{~d}$ & 33,3 & $\mathrm{~b}$ & 0,0 & $\mathrm{a}$ & 2,8 & $\mathrm{a}$ \\
\hline L3P9 & 44,4 & $\mathrm{~b}$ & 87,8 & $\mathrm{~d}$ & 16,1 & $\mathrm{a}$ & 3,3 & $\mathrm{a}$ & 6,7 & $\mathrm{~b}$ \\
\hline L2P4 & 46,7 & $\mathrm{~b}$ & 20,0 & $\mathrm{~b}$ & 53,3 & $\mathrm{~b}$ & 6,7 & $\mathrm{a}$ & 0,0 & $\mathrm{a}$ \\
\hline L4P13 & 46,7 & $\mathrm{~b}$ & 50,0 & $\mathrm{c}$ & 36,7 & $\mathrm{~b}$ & 10,0 & $\mathrm{a}$ & 6,7 & $\mathrm{~b}$ \\
\hline Monte Alto - Branca & 48,5 & $\mathrm{~b}$ & 70,9 & $\mathrm{~d}$ & 16,1 & $\mathrm{a}$ & 3,3 & $\mathrm{a}$ & 12,7 & $\mathrm{~b}$ \\
\hline Kumagai Branca & 48,9 & $\mathrm{~b}$ & 65,9 & $\mathrm{~d}$ & 13,3 & $\mathrm{a}$ & 0,0 & $\mathrm{a}$ & 6,7 & $\mathrm{~b}$ \\
\hline L3P12 & 50,0 & $\mathrm{~b}$ & 6,7 & $\mathrm{a}$ & 7,5 & $\mathrm{a}$ & 3,3 & $\mathrm{a}$ & 0,0 & $\mathrm{a}$ \\
\hline L8P32A & 50,0 & $\mathrm{~b}$ & 26,7 & $\mathrm{~b}$ & 43,3 & $\mathrm{~b}$ & 3,3 & $\mathrm{a}$ & 6,7 & $\mathrm{~b}$ \\
\hline Rubi Supreme & 51,5 & $\mathrm{~b}$ & 34,1 & $\mathrm{~b}$ & 32,2 & $\mathrm{~b}$ & 3,3 & $\mathrm{a}$ & 3,3 & $\mathrm{a}$ \\
\hline L5P20 & 53,3 & $\mathrm{~b}$ & 56,7 & $\mathrm{c}$ & 20,0 & $\mathrm{a}$ & 3,3 & $\mathrm{a}$ & 13,3 & $\mathrm{~b}$ \\
\hline L2P6 & 53,3 & $\mathrm{~b}$ & 73,3 & $\mathrm{~d}$ & 16,7 & $\mathrm{a}$ & 6,7 & $\mathrm{a}$ & 0,0 & $\mathrm{a}$ \\
\hline FAO - Unesp & 56,7 & $\mathrm{c}$ & 13,3 & $\mathrm{a}$ & 16,7 & $\mathrm{a}$ & 0,0 & $\mathrm{a}$ & 6,7 & $\mathrm{~b}$ \\
\hline L8P32B & 60,0 & $\mathrm{c}$ & 83,3 & $\mathrm{~d}$ & 26,7 & $\mathrm{~b}$ & 6,7 & $\mathrm{a}$ & 10,0 & $\mathrm{~b}$ \\
\hline Indiana - Unesp & 61,2 & $\mathrm{c}$ & 26,1 & $\mathrm{~b}$ & 23,0 & $\mathrm{~b}$ & 0,0 & $\mathrm{a}$ & 3,3 & $\mathrm{a}$ \\
\hline L5P18 & 61,5 & $\mathrm{c}$ & 58,8 & $\mathrm{c}$ & 25,8 & $\mathrm{~b}$ & 0,0 & $\mathrm{a}$ & 0,0 & $\mathrm{a}$ \\
\hline Webber Supreme & 63,0 & $\mathrm{c}$ & 0,0 & $\mathrm{a}$ & 22,2 & $\mathrm{a}$ & 0,0 & $\mathrm{a}$ & 3,7 & $\mathrm{a}$ \\
\hline Rica - J-2 & 64,4 & $\mathrm{c}$ & 14,1 & $\mathrm{~b}$ & 30,0 & $\mathrm{~b}$ & 0,0 & $\mathrm{a}$ & 0,0 & $\mathrm{a}$ \\
\hline Ogawa1 & 65,8 & $\mathrm{c}$ & 31,7 & $\mathrm{~b}$ & 13,3 & $\mathrm{a}$ & 0,0 & $\mathrm{a}$ & 0,0 & $\mathrm{a}$ \\
\hline L5P21 & 67,3 & $\mathrm{c}$ & 3,3 & $\mathrm{a}$ & 13,3 & $\mathrm{a}$ & 3,3 & $\mathrm{a}$ & 0,0 & $\mathrm{a}$ \\
\hline L4P17 & 68,3 & $\mathrm{c}$ & 39,2 & $\mathrm{c}$ & 17,5 & $\mathrm{a}$ & 0,0 & $\mathrm{a}$ & 3,3 & $\mathrm{a}$ \\
\hline L6P22 & 68,5 & $\mathrm{c}$ & 7,0 & $\mathrm{a}$ & 23,3 & $\mathrm{~b}$ & 10,0 & $\mathrm{a}$ & 0,0 & $\mathrm{a}$ \\
\hline L1P3 & 73,3 & $\mathrm{c}$ & 26,7 & $\mathrm{~b}$ & 25,0 & $\mathrm{~b}$ & 0,0 & $\mathrm{a}$ & 0,0 & $\mathrm{a}$ \\
\hline L6P23 & 73,9 & $\mathrm{c}$ & 54,8 & $\mathrm{c}$ & 6,4 & $\mathrm{a}$ & 6,7 & $\mathrm{a}$ & 9,7 & $\mathrm{~b}$ \\
\hline L8P31 & 76,7 & $\mathrm{c}$ & 53,3 & $\mathrm{c}$ & 16,7 & $\mathrm{a}$ & 6,7 & $\mathrm{a}$ & 0,0 & $\mathrm{a}$ \\
\hline Kioshi2 & 77,3 & $\mathrm{c}$ & 77,9 & d & 12,7 & $\mathrm{a}$ & 3,3 & $\mathrm{a}$ & 3,3 & $\mathrm{a}$ \\
\hline L3P7 & 77,8 & $\mathrm{c}$ & 27,8 & $\mathrm{~b}$ & 36,1 & $\mathrm{~b}$ & 8,3 & $\mathrm{a}$ & 2,8 & $\mathrm{a}$ \\
\hline Creme Arredondada (Unesp) & 79,0 & $\mathrm{c}$ & 65,7 & $\mathrm{~d}$ & 41,0 & $\mathrm{~b}$ & 11,4 & $\mathrm{a}$ & 0,0 & $\mathrm{a}$ \\
\hline L3P10 & 83,9 & $\mathrm{~d}$ & 67,9 & $\mathrm{~d}$ & 13,3 & $\mathrm{a}$ & 0,0 & a & 0,0 & $\mathrm{a}$ \\
\hline Vermelha Perfumada & 84,5 & $\mathrm{~d}$ & 64,2 & $\mathrm{~d}$ & 12,4 & $\mathrm{a}$ & 3,3 & $\mathrm{a}$ & 0,0 & $\mathrm{a}$ \\
\hline Goiaba Polpa Amarela & 85,9 & d & 65,6 & $\mathrm{~d}$ & 27,4 & $\mathrm{~b}$ & 6,7 & a & 0,0 & $\mathrm{a}$ \\
\hline L4P14 & 86,3 & d & 48,1 & $\mathrm{c}$ & 7,0 & $\mathrm{a}$ & 0,0 & $\mathrm{a}$ & 20,4 & $\mathrm{~b}$ \\
\hline L4P15 & 87,3 & d & 74,5 & $\mathrm{~d}$ & 16,4 & $\mathrm{a}$ & 3,3 & $\mathrm{a}$ & 0,0 & $\mathrm{a}$ \\
\hline Ogawa3 & 87,5 & d & 25,0 & $\mathrm{~b}$ & 25,0 & $\mathrm{~b}$ & 0,0 & $\mathrm{a}$ & 0,0 & $\mathrm{a}$ \\
\hline L5P19 & 90,3 & d & 87,3 & $\mathrm{~d}$ & 9,7 & $\mathrm{a}$ & 3,0 & $\mathrm{a}$ & 0,0 & $\mathrm{a}$ \\
\hline L2P5 & 90,6 & d & 73,6 & $\mathrm{~d}$ & 25,8 & $\mathrm{~b}$ & 0,0 & $\mathrm{a}$ & 0,0 & $\mathrm{a}$ \\
\hline IAC-4 - Cica & 96,7 & d & 90,0 & $\mathrm{~d}$ & 20,0 & $\mathrm{a}$ & 3,3 & $\mathrm{a}$ & 0,0 & $\mathrm{a}$ \\
\hline Kioshi1 & 96,7 & d & 96,7 & d & 23,3 & $\mathrm{~b}$ & 0,0 & $\mathrm{a}$ & 0,0 & $\mathrm{a}$ \\
\hline Campos & 100,0 & d & 83,3 & d & 23,3 & $\mathrm{~b}$ & 0,0 & $\mathrm{a}$ & 0,0 & $\mathrm{a}$ \\
\hline L1P2 & 100,0 & $\mathrm{~d}$ & 70,6 & $\mathrm{~d}$ & 35,5 & $\mathrm{~b}$ & 0,0 & $\mathrm{a}$ & 0,0 & $\mathrm{a}$ \\
\hline Taquaritinga Comum & 100,0 & $\mathrm{~d}$ & 53,3 & $\mathrm{c}$ & 13,3 & $\mathrm{a}$ & 6,7 & $\mathrm{a}$ & 3,3 & $\mathrm{a}$ \\
\hline $\mathrm{F}$ (acessos) & \multicolumn{2}{|c|}{$6,7^{* *}$} & \multicolumn{2}{|c|}{$12,3 * *$} & \multicolumn{2}{|c|}{$1,7^{*}$} & \multicolumn{2}{|c|}{$1,8 * *$} & \multicolumn{2}{|c|}{$2,2 * *$} \\
\hline CV (\%) & \multicolumn{2}{|c|}{11,9} & \multicolumn{2}{|c|}{12,8} & \multicolumn{2}{|c|}{22,8} & \multicolumn{2}{|c|}{19,1} & \multicolumn{2}{|c|}{18,2} \\
\hline
\end{tabular}


incidências inferiores a $14 \%$. O acesso Webber Supreme teve completa ausência de sintomas de pinta-preta (Tabela 2).

Já para a podridão peduncular, os acessos significativamente menos afetados foram: L6P23, L4P14, L3P12, Monte Alto - Comum 1, L5P19, Vermelha Perfumada, Kioshi 2, L5P21, Ogawa 1, Taquaritinga Comum, Kumagai Branca, L3P10, IAC-4 - Unesp, Monte Alto - Branca, L3P9, L4P15, FAO - Unesp, L8P31, L2P6, L4P17, Vermelha Redonda (Shimoda), L5P20, IAC-4 - Cica, Webber Supreme e Kioshi 3, com incidências variando entre $6,4 \%$ e $22,2 \%$ (Tabela 2 ).

Os patógenos identificados causando sintomas de podridão peduncular foram: Fusicoccum sp. (85,2 \%), Phomopsis sp. (10,7 \%), Phoma sp. (3,3\%), Dothiorella sp. (0,4\%) e Lasiodiplodia sp. (0,4\%). Segundo Soares-Colletti et al. (2014), a podridão peduncular causada por Fusicoccum atingiu incidência média de $23,7 \%$, em goiabas 'Kumagai' comercializadas na Ceagesp e Ceasa-Campinas, após 10 dias de armazenamento a $25^{\circ} \mathrm{C}$, não diferindo, significativamente, da antracnose $\mathrm{e}$ da pinta preta.

Os acessos Vermelha Redonda (Shimoda), L5P21, L3P12, EEF-3, IAC-4 - Unesp e Monte Alto - Comum 1 destacaram-se dos demais por apresentarem as menores incidências em pelo menos duas doenças, enquanto os acessos L4P13, L1P2 e Creme Arredondada (Unesp) foram altamente suscetíveis às três doenças (antracnose, pinta preta e podridão peduncular), com incidências superiores a $35 \%$ (Tabela 2).

As podridões causadas por Pestalotia e Mucor foram, de maneira geral, menos incidentes. Não houve diferenças entre os acessos para podridão por Pestalotia. Já a podridão por Mucor incidiu de maneira significativamente diferente, sendo os acessos L4P16, Vermelha Redonda (Shimoda), Monte Alto - Branca, L5P20 e L4P14 os mais afetados, com incidências superiores a $10 \%$.

Estudos de resistência de acessos de goiabeira a doenças já foram realizados para bacteriose, ferrugem da goiabeira e nematoide das galhas (Piccinin et al. 2005, Rezende 2006, Martins et al. 2013). Contudo, este é o primeiro estudo de acessos de goiabeira visando a avaliar o comportamento destes, em relação às doenças pós-colheita. $\mathrm{O}$ emprego de variedades resistentes é considerado o método ideal de manejo, por ser aplicável a grandes áreas e possuir

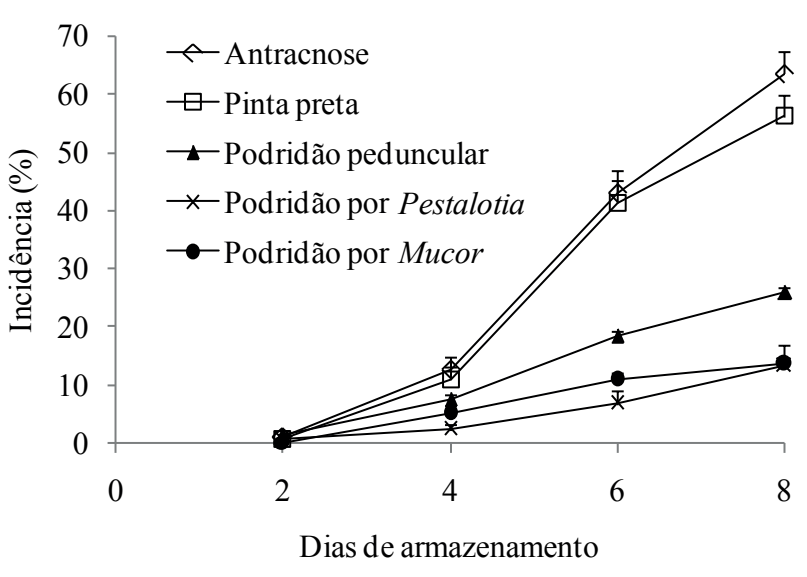

Figura 1. Progresso na incidência das doenças pós-colheita em goiabas (média dos acessos doentes), durante oito dias de armazenamento dos frutos a $25 \pm 1{ }^{\circ} \mathrm{C}$ e $70-80 \%$ de umidade relativa. As barras representam o erro padrão da média (Bauru, SP, 2014).

baixo impacto ambiental, se comparado à utilização de defensivos agrícolas.

A incidência das doenças aos dois dias ficou abaixo de $2 \%$. Diferenças entre as doenças foram observadas a partir do quarto dia, com maiores incidências de antracnose e pinta preta, que apresentaram mais de $30 \%$ de incidência aos seis dias. Ao final do armazenamento, a antracnose foi a doença mais incidente $(63,3 \%)$, seguida da pinta preta $(56,2 \%)$ e podridão peduncular (26,0 \%) (Figura 1).

A goiaba é um fruto de alta perecibilidade e os danos em pós-colheita podem chegar a $40 \%$, a partir do terceiro dia de armazenamento, em função, principalmente, da ocorrência de doenças (Fischer et al. 2011). Os resultados observados no presente estudo estão de acordo com os relatados por Barkai-Golan (2001) e Fischer et al. (2011), os quais também demonstram que a antracnose e a pinta preta são as doenças pós-colheita mais frequentes em frutos de goiabeira.

\section{CONCLUSÕES}

1. A antracnose e a pinta preta são as doenças pós-colheita mais frequentes nos frutos de goiabeira.

2. Os acessos Vermelha Redonda (Shimoda), L5P21, L3P12, EEF-3, IAC-4 - Unesp e Monte Alto - Comum 1 destacam-se dos demais por apresentarem as menores incidências de antracnose e pinta preta.

3. Os acessos L4P13, L1P2 e Creme Arredondada (Unesp) são altamente suscetíveis à antracnose, pinta preta e podridão peduncular. 
4. Os patógenos Fusicoccum sp., Phomopsis sp., Phoma sp., Dothiorella sp. e Lasiodiplodia sp. isolados de frutos de goiabeira causam sintomas de podridão peduncular.

\section{AGRADECIMENTOS}

À Fundação de Apoio à Pesquisa do Estado de São Paulo (Fapesp), pelo auxílio financeiro na condução deste trabalho (Processo 2012/03807-0).

\section{REFERÊNCIAS}

AMORIM, L. et al. Epidemiologia e manejo de doenças pós-colheita de goiabas. In: NATALE, W. et al. Cultura da goiaba: do plantio à comercialização. Jaboticabal: FCAVUnesp, 2009. p. 309-326.

BARKAI-GOLAN, R. Postharvest diseases of fruits and vegetables: development and control. Amsterdam: Elsevier, 2001.

BARNETT, H. L.; HUNTER, B. B. Illustrated genera of imperfect fungi. 4. ed. Saint Paul: American Phytopathology Society Press, 1998.

CAMPBELL, C. D.; MADDEN, L. V. Introduction to plant disease epidemiology. New York: John Willey, 1990.

FISCHER, I. H. et al. Danos em pós-colheita de goiabas na região do centro-oeste paulista. Bragantia, Campinas, v. 70 , n. 3, p. 570-576, 2011.

JUNQUEIRA, N. T. V.; COSTA, H. Controle das doenças da goiabeira. In: ZAMBOLIM, L. et al. Controle de doenças de plantas: fruteiras. Viçosa: UFV, 2002. p. 12471277.

MANICA, I. et al. Fruticultura tropical: goiaba. Porto Alegre: Cinco Continentes, 2000.

MARTINS, L. S. S. et al. Parasitismo de Meloidogyne enterolobii em espécies de myrtaceae. Revista Brasileira de Fruticultura, Jaboticabal, v. 35, n. 2, p. 477-484, 2013.

PICCININ, E. et al. Doenças da goiabeira (Psidium guajava L.). In: KIMATI, H. et al. Manual de fitopatologia: doenças das plantas cultivadas. 4. ed. São Paulo: Agronômica Ceres, 2005. p. 401-405.

PINHEIRO, S. S. C. Qualidade de goiabas ensacadas e manejadas com diferentes produtos fitossanitários, sob manejo orgânico. 2006. 106 f. Tese (Doutorado em Fitotecnia) - Universidade Federal de Viçosa, Viçosa, 2006.

REZENDE, A. M. F. A. Estudo sobre a resistência genética e produtos químicos no controle da bacteriose da goiabeira. 2006. 84 f. Dissertação (Mestrado em Fitopatologia) - Universidade de Brasília, Brasília, DF, 2006.

SOARES-COLLETTI, A. R.; FISCHER, I. H.; LOURENÇO, S. A. Incidence of postharvest diseases on 'Kumagai' and 'Pedro Sato' guavas at wholesale markets in Brazil. Tropical Plant Pathology, Brasília, DF, v. 39, n. 6, p. 478-482, 2014.

TOZETTO, L. J.; RIBEIRO, W. R. C. Ocorrência de podridão de frutos de goiaba (Psidium guajava) causada por Phyllosticta sp. em Brasília, DF. Fitopatologia Brasileira, Brasília, DF, v. 18, supl., p. 160, 1993. 